\title{
The Origin of the Decoupling of Oxygen and Silicon Dynamics in Liquid Silica as Expressed by its Potential Energy Landscape
}

\author{
A. Saksaengwijit and A. Heuer \\ Westfälische Wilhelms-Universität Münster, \\ Institut für Physikalische Chemie \\ and International Graduate School of Chemistry \\ Corrensstr. 30, 48149 Münster, Germany
}

(Dated: May 22, 2018)

\begin{abstract}
The oxygen and silicon dynamics in silica is compared via computer simulations. In agreement with experimental data and previous simulations a decoupling of oxygen and silicon dynamics is observed upon cooling. The origin of this decoupling is studied in the framework of the potential energy landscape. From analysis of the transition features between neighboring superstructures of minima, denoted metabasins, the differences between the oxygen and the silicon dynamics can be quantified. The decoupling can be explicitly related to the presence of generalized rotational processes, giving rise to oxygen but not to silicon displacement. Closer analysis of these processes yields important insight into the nature of the potential energy landscape of silica. The physical picture of relaxation processes in silica, obtained in previous work for the oxygen dynamics, is consistent with the decoupling effects, elucidated here.
\end{abstract}

PACS numbers: 64.70.Pf,65.40.Gr,66.20.+d

\section{INTRODUCTION}

Silica is a well-known prototype network glass former. It is classified as a strong liquid in the Angell scheme 1 because the temperature dependence of its dynamics displays an Arrhenius behavior. Interestingly, oxygen and silicon dynamics are characterized by different activation energies. Close to the glass transition temperature $T_{g}$ the difference between the self-diffusion constants for oxygen $D_{O}$ and silicon $D_{S i}$ is nearly two orders of magnitude [2, 3]. Moreover, it has been shown that the viscous dynamics of liquid silica close to $T_{g}$ is strongly controlled by the silicon dynamics 4]. The large difference in diffusivity indicates different relaxation mechanisms for oxygen and silicon atoms in liquid silica at low temperatures.

The decoupling of silicon and oxygen single-particle dynamics has been already studied via computer simulations of $\mathrm{SiO}_{2}$ [5] modelled by the BKS potential 6]. It turned out that in the limit of very high temperatures the diffusive dynamics of oxygen and silicon is similar $\left(D_{O} / D_{S i} \approx 1.3\right)$. Upon cooling the slowdown in silicon dynamics is significantly stronger as compared to that in oxygen dynamics. This results in a faster decrease of $D_{S i}$ at low temperatures, e.g at $2800 \mathrm{~K}$ one has $D_{O} / D_{S i} \approx 2.3$ [5]. This has been regarded as an evidence for a decoupling dynamics. Interestingly, the temperature dependence of the average time-scale for the lifetime of Si-O bonds follows $D_{O}(T)$ rather than $D_{S i}(T)$ [5]. Furthermore, the self part of the van Hove correlation function for oxygen displays a clear additional peak, which corresponds to the hopping dynamics. In contrast, no clear hopping peak is present for the case of silicon 5]. Moreover, the recent study on the facilitated dynamics in liquid silica [7, 8] shows that the cooperative motion for oxygen dynamics can be described by a string-like motion [9] whereas the usual string-like motion is absent for the case of silicon atoms.

The potential energy landscape (PEL) has been shown to be an indispensable tool to elucidate the nature of the dynamics and thermodynamics of liquids 10, 11, 12, 13, $14,15,16,17,18$. In this framework the dynamics of the system is represented by the dynamics of a point in the high dimensional configurational space [19]. The thermodynamics of the system can be expressed in terms of the statistical properties of the inherent structures (IS), i.e. the local minima of the PEL [14, 16, 20]. Naturally, the essential features observed in the small subsystem are trivially averaged out on a macroscopic level [21, 22]. Therefore, to understand the decoupling dynamics in terms of the PEL properties it is important to analyze sufficiently small systems without relevant finitesize effects.

Recent studies on the thermodynamics of this model have shown that the distribution of IS energies $G(e)$ possesses a low-energy cutoff $e_{c}$ [22]. Below $e_{c}$ the configurational energy is strongly depleted as compared to the Gaussian distribution, which describes $G(e)$ for $e>e_{c}$. An important step to characterize the dynamics has been the introduction of metabasins (MB) [12, 23]. This corresponds to a coarse-graining of the configuration space. Groups of minima between which the system performs forward and backward jumps are regarded as single states, i.e MB. The total dynamics can then be formally described as a sequence of jumps between MB, each characterized by a waiting time $\tau$ and an energy $e$ which is chosen as the minimum IS energy of the corresponding group of IS. Actually, the distribution $G(e)$ of $\mathrm{MB}$ energies is virtually identical to that of IS such that the thermodynamics does not change when switching from the IS to the MB description. It turns out for silica that on this level of description the oxygen dynamics of the system can be (to a good approximation) char- 
acterized as a random-walk in configuration space with a temperature-independent average hopping distance [23]. This provides a strong link between long-range transport and microscopic dynamics. As an immediate consequence the temperature dependence of $D_{O}(T)$ is determined by the temperature dependence of the average waiting time. Furthermore, it has been shown that the diffusion and relaxation in liquid silica has been related to the defect structure 23, 24, 25] since close to defects the mobility is largely enhanced.

The goal of this paper is twofold. First, we want to show why the decoupling of oxygen and silicon dynamics as well as the proportionality of the oxygen diffusion with the inverse bond lifetime is a natural consequence of the nature of the dynamics of BKS-silica. It will turn out that it is the presence of rotational displacements which are the origin of the decoupling. Second, we will relate the occurrence of these specific displacements to the properties of the PEL. This will be another example where the reference to properties of the PEL gives new insight into the complex dynamics of supercooled liquids.

The outline is as follows. In Sect.II we present some computational details. In Sect.III we show a detailed analysis of the nature of the MB transitions. In this way the relevance of rotational processes is elucidated. Based on these results we can unambiguously explain the decoupling of the oxygen and silicon diffusion in terms of these processes, as shown in Sect.IV. In Sect.V the rotational processes are related to properties of the PEL. We close with a discussion and a summary in Sect.VI. In particular, we show that the general physical picture of the relaxation processes in silica, as derived in our previous work [23], is fully compatible with the results, obtained for the relation of the oxygen and silicon dynamics in this work.

\section{TECHNICAL DETAILS}

We have performed molecular dynamics simulations of liquid silica at constant volume and energy (microcanonical ensemble). The density of liquid silica is $\rho=2.30 \mathrm{gcm}^{-3}$, which is similar to that in previous simulations [5, 13. The standard BKS potential [6] is used to describe the interaction energy for liquid silica. The Coulombic interaction is calculated with the Ewald-summation technique. The short range interaction is truncated and shifted to zero at the cutoff radius $r_{c}$. To avoid an energy drift $r_{c}$ is set to $8.5 \AA$, which is slightly larger than the one used previously [5]. In this work we analyze the temperature range from $2800 \mathrm{~K}$ to $4000 \mathrm{~K}$, using well-equilibrated samples. All results have been averaged over three independent runs in order to gain better statistics.

It has been shown that for BKS-silica a system size of approx. 100 particles with periodic boundary conditions is sufficient to avoid relevant finite-size effects for the configurational entropy, the relaxation dynamics of
BKS-silica (in terms of the diffusive activation energy and the degree of non-exponentiality in the incoherent scattering function) in the accessible temperature range and the properties of tunneling systems [22, 23, 24, 26]. As in our previous work we use a system size of $N=99$. To characterize the distance between two configurations we use the displacement of the oxygen atoms if not mentioned otherwise.

For each equilibrium run, the system is quenched via the standard conjugated-gradient method. The times for the quench have been optimized via the bisectioning method to enable an efficient search for the precise location of the metabasin transitions [12]. In this way we obtain a time series of IS energies, which contains all relevant IS transitions which are necessary to characterize all MB transitions. As mentioned above MB are characterized by a waiting time $\tau$ and an energy $e$. The energy of a MB is defined as the lowest IS energy in a MB (see 27] for a precise definition of MB).

For later purposes we introduce two different energy distributions, relevant at temperature $T\left(=1 /\left(k_{B} \beta\right)\right)$.

$$
p(e, T) \propto G(e) \exp (-\beta e)
$$

is the Boltzmann weighted distribution of MB energies at temperature $T$ [28]. It expresses the probability that at a randomly given time the system is in a $\mathrm{MB}$ of energy e. Furthermore, we define

$$
\varphi(e, T)=\frac{p(e, T)\langle\tau(T)\rangle}{\langle\tau(e, T)\rangle}
$$

where $\langle\tau(e, T)\rangle$ is the average waiting time of a MB with energy $e . \varphi(e, T)$ denotes the probability that after a $\mathrm{MB}$ transition the new MB has the energy $e$ [12].

\section{DYNAMIC PROPERTIES OF OXYGEN AND SILICON ATOMS}

\section{A. Metabasin analysis}

In the previous studies on the binary Lennard-Jones system (BMLJ) it has been shown that the mean square displacement $R^{2}\left(n_{M B}\right)$ after $n_{M B}$ transitions between MB is independent of temperature except for extremely high temperatures for which minima of the PEL are not relevant due to the dominance of anharmonic effects 12. As a consequence the diffusion constant $D(T)$ is related to the average $\mathrm{MB}$ waiting time $\langle\tau(T)\rangle$ via a temperature-independent jump length $d$, i.e.

$$
D(T)=d^{2} / 6 N\langle\tau(T)\rangle .
$$

Here we repeat this analysis for the oxygen and silicon dynamics in silica by calculating $R_{O}^{2}\left(n_{M B}\right)$ and $R_{S i}^{2}\left(n_{M B}\right)$, respectively; see Fig प One directly observes that $R_{O}^{2}\left(n_{M B}\right)$ is temperature-independent whereas there is a significant and systematic temperature shift 


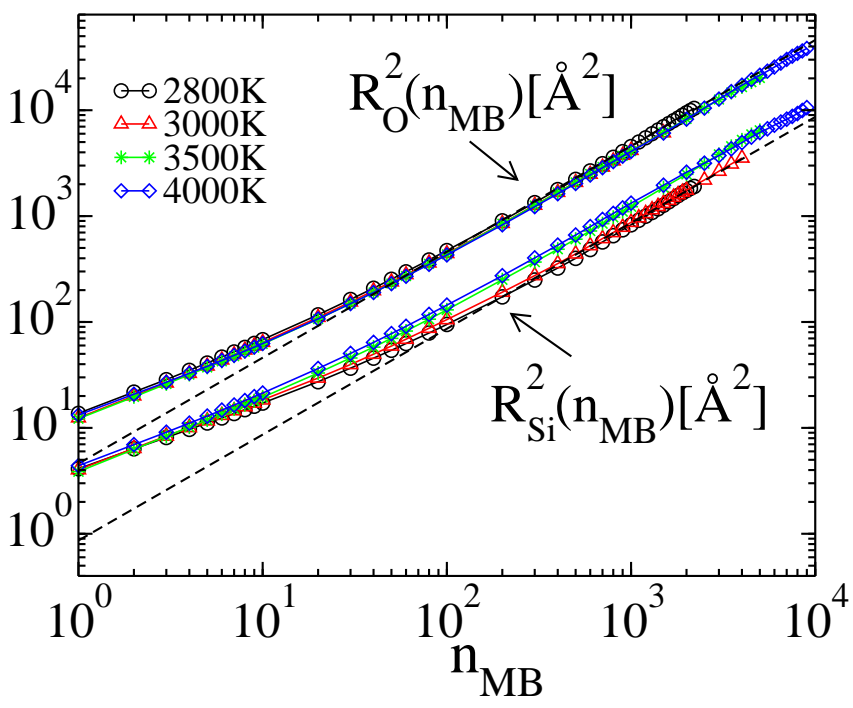

Figure 1: The average mean square displacement of oxygen and silicon after $n \mathrm{MB}$ transitions, i.e. $R_{O}^{2}\left(n_{M B}\right)$ and $R_{S i}^{2}\left(n_{M B}\right)$. The value of $n_{M B}$ indicates the number of $\mathrm{MB}$ transitions. For the weak but significant temperature dependence of $R_{S i}^{2}\left(n_{M B}\right) / n_{M B}$ in the constant regime for large $n_{M B}$ one obtains $1.3 \AA^{2}, 1.2 \AA^{2}, 0.9 \AA^{2}$ and $0.8 \AA^{2}$ for the four temperatures (starting from high temperatures), respectively.

of $R_{S i}^{2}\left(n_{M B}\right)$. Actually, the temperature independence of $R_{O}^{2}\left(n_{M B}\right)$ has been already used in our previous work [23. to justify the use of Eq[3 also for silica. Of course, the decoupling of oxygen and silicon directly follows from the different behavior of $R_{O}^{2}\left(n_{M B}\right)$ and $R_{S i}^{2}\left(n_{M B}\right)$.

On a qualitative level these observations agree with the previous analysis of the decoupling of oxygen and silicon [5]. By analyzing the time decay $\tau_{B}$ of the Si$\mathrm{O}$ bond residual time, Horbach and Kob observed that $\tau_{B} \cdot D_{O}$ does not depend on temperature whereas $\tau_{B} \cdot D_{S i}$ shows a significant temperature dependence at low temperatures. $\tau_{B}$ should be proportional to the waiting time of MB $\tau_{M B}$ because typical MB transitions will involve bond-breaking events [23]. Thus the previous observations $D_{O}^{-1} \propto \tau_{B}$ but $D_{S i}^{-1} \not \tau_{B}$ are indeed qualitatively equivalent to the results in Fig 1

But how to rationalize the temperature dependence of $R_{S i}^{2}\left(n_{M B}\right)$ ? The most simple explanation would be that typical silicon displacements during MB transitions are smaller at lower temperatures. In this case the temperature dependence would reflect a change of the jump mechanism with temperature. In literature, the complicated relaxation of silicon atoms has been related to the possible presence of deep potential traps for silicon [5]. In this scenario, however, one would expect that there is also a systematic temperature dependence for, e.g. $R_{S i}^{2}\left(n_{M B}=1\right)$, which corresponds to the typical displacement after one MB transition. Interestingly, in our simulations we have not found such a temperature dependence; see Fig 1

\section{B. Rotational and translational periods}

Here we show that a different scenario is responsible for the observations, shown in Fig 1 For each time series of IS (MB) at different temperatures, we have observed some rotational processes. More precisely this means that during the time evolution the system is found in different configurations which are identical except for a permutation of oxygen atoms. The most simple realization of rotational processes are rotations of a $\mathrm{SiO}_{4}$ tetrahedral unit along the three-fold $\left(\mathrm{C}_{3}\right)$ or two-fold $\left(\mathrm{C}_{2}\right)$ axis but also more complex permutations have been observed.

To elucidate this possibility in a quantitative way we first define rotational periods as the maximum time intervals $\left[t_{1}, t_{2}\right]$ with the property that the configurations at $t_{1}$ and $t_{2}$ are identical except for the permutation of oxygen atoms. Of course, during one rotational period several rotations may take place. To characterize the dynamics during a rotational period further one can identify the different oxygen permutations. They are denoted rotational states. Naturally, each rotational period contains at least two rotational states but also more may be present.

In Fig 2] a sequence of IS is shown. The different rotational states which are visited during this time period are indicated. Note that within the rotational period the system may escape from a rotational state and either come back to the same state or end up in another permutation of oxygen atoms. Typically, subsequent rotational states are not connected by a single saddle but rather by highenergy IS. We note in passing that for all simulation runs we have not observed any permutation of silicon atoms. The time intervals between the rotational periods are denoted translational periods; see Fig[2] Whereas the contributions from the long-time dynamics of oxygen atoms come from rotational and translational periods, the longtime dynamics of silicon atoms is exclusively related to the translational periods.

On average, many IS transitions are required for the system to go from one rotational state to another; see Fig 2] During this time also the silicon atoms display significant motion but finally go back to the original position. From a single-atom perspective during rotational processes the position of some oxygen atoms change. Thus, rotational processes contribute to the long-range dynamics of oxygen as opposed to that of silicon. The simulation data show that rotational periods become more relevant at low temperatures (see below for a quantification of this statement). Thus, the fraction of MB transitions which finally are irrelevant for the silicon dynamics increases with decreasing temperature. From this observation it is indeed expected that $R_{S i}^{2}\left(n_{M B}\right)$ is smaller at lower temperatures as observed in Fig 1 Furthermore, a much weaker temperature dependence should occur for $R_{S i}^{2}\left(n_{M B}=1\right)$ because on the single-transition scale a silicon atom hardly realizes that a few MB-transitions later it will go back to the original configuration. Stated differently, the strong forward- 


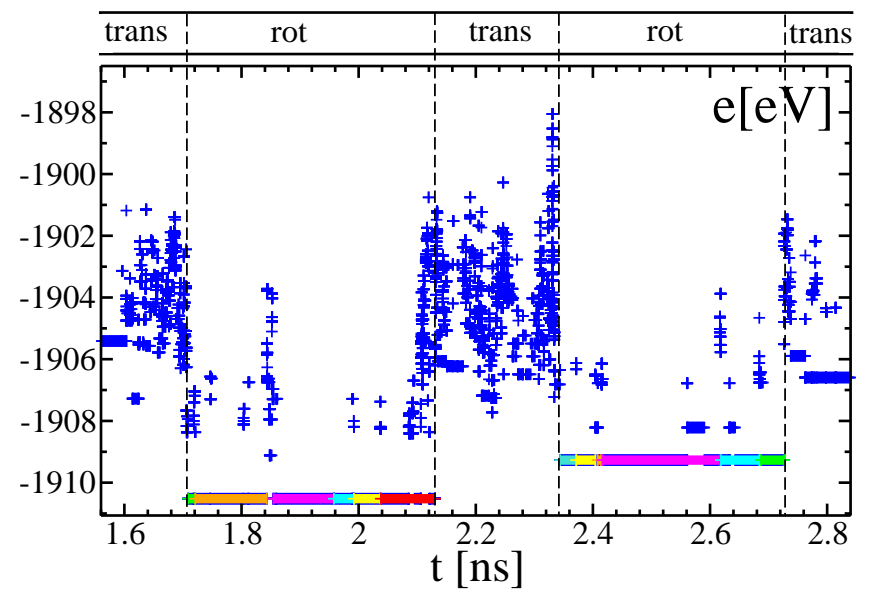

Figure 2: The time series of IS energies at 3500K. The translational and rotational periods are labeled by trans and rot, respectively.

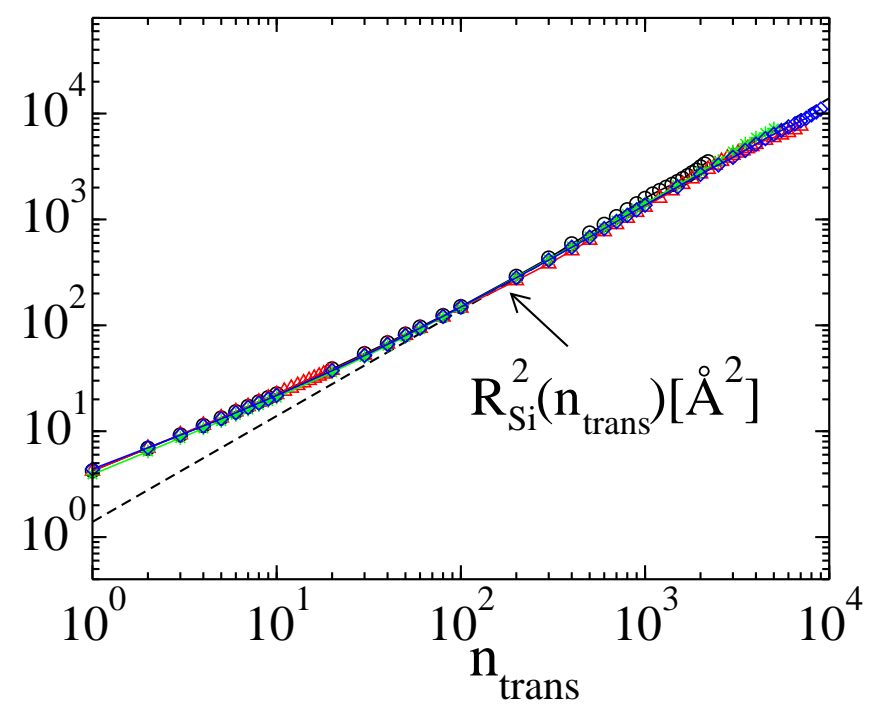

Figure 3: The average mean square displacement of silicon $R_{M B, S i}^{2}(n)$ after $n \mathrm{MB}$ transitions. In contrast to Fig. 1 only MB transitions within the translational periods are taken into account.

backward correlations of the silicon dynamics during the rotational periods should show up by a stronger subdiffusive behavior in $R_{S i}^{2}\left(n_{M B}\right)$ in agreement with Fig 1

The hypothesis that the slowing-down of silicon is exclusively due to the presence of rotational periods can now easily be tested. For this purpose we have calculated $R_{S i}^{2}\left(n_{\text {trans }}\right)$ by ignoring the $\mathrm{MB}$ transitions during the rotational periods. Thus for this analysis we only count MB transitions during the translational periods, as indicated by the index trans. The result is shown in Fig 3. Within statistical uncertainties one observes a temperature-independent behavior. This unambiguously shows that the decoupling of oxygen and silicon dynamics is exclusively due to the increasing relevance of rotational processes at lower temperatures. Furthermore, the non-

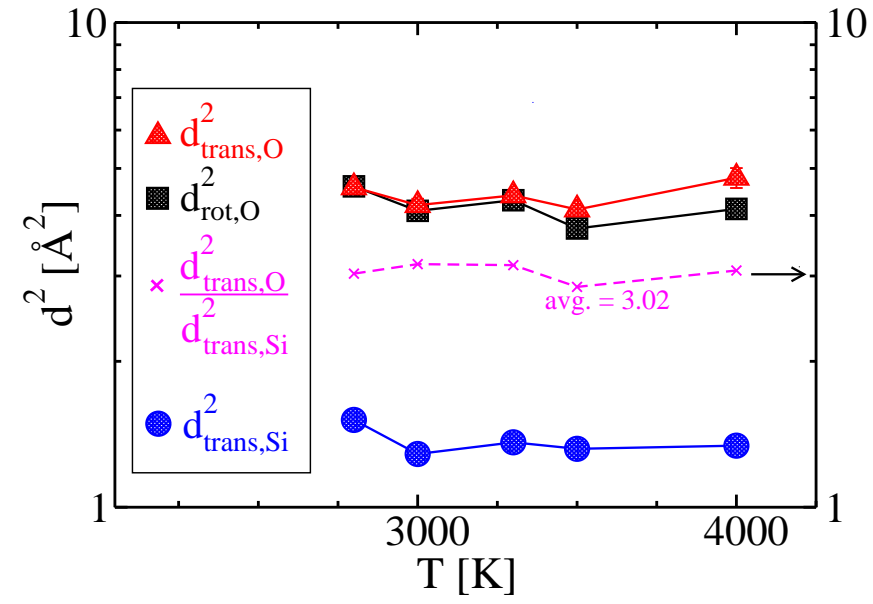

Figure 4: Temperature dependence of the rotational and translational spatial factors $d_{\text {trans, },}^{2}(T), d_{\text {trans }, S i}^{2}(T)$ and $d_{\text {rot }, O}^{2}$ for MB transitions (see definitions in text).

diffusive part for small $n_{\text {trans }}$, reflecting deviations from a pure random-walk behavior, becomes smaller and similar in size to that of the oxygen dynamics in Fig 1 From the large- $\mathrm{n}_{\text {trans }}$ behavior of $R_{S i}^{2}\left(n_{\text {trans }}\right) \propto n_{\text {trans }}$ the proportionality constant can be interpreted as an effective jump-length $d_{\text {trans,Si }}^{2}$. It is shown in Fig 4 As expected, no relevant temperature dependence is observed.

Is the oxygen dynamics different in rotational as compared to translational periods? Starting with the oxygen dynamics during rotational periods we first determine the number of MB transitions $n_{r o t, i}$ and the squared displacement $d_{i}^{2}$ during the $\mathrm{i}$-th rotational period. Formally, $d_{i}^{2}$ is the squared Euclidian distance between the first and the last configuration of a rotational period. Then one can define the effective oxygen hopping distance $d_{r o t, O}^{2}$ during rotational periods via

$$
d_{\text {rot }, O}^{2} \equiv \frac{\sum_{i} d_{i}^{2}}{\sum n_{\text {rot }, i}} .
$$

Its temperature dependence is also shown in Fig 4 Again, within statistical uncertainties no temperature dependence is observed.

Formally, the total displacement of the oxygen atoms during the translational periods can be obtained from the total mean square displacement during the whole simulation run minus the contributions from the rotational periods, i.e. $\sum_{i} d_{i}^{2}$. For the number of MB transitions $n_{\text {trans }}$ in the translational periods one simply has $n_{\text {trans }}=n_{M B}-n_{\text {rot }}$. In analogy to Eq廿 one can define $d_{\text {trans }, O}^{2}$ as the effective hopping distance during the translational periods. Again, as seen in Fig 4 also this observable does not display any significant temperature dependence.

The ratio of $d_{\text {trans }, O}^{2} / d_{\text {trans }, S i}^{2}$ is close to three which is larger than the statistical factor of 2 . Thus, the silicon dynamics is indeed somewhat more hindered than the oxygen dynamics. This reduction in mobility, however, is 


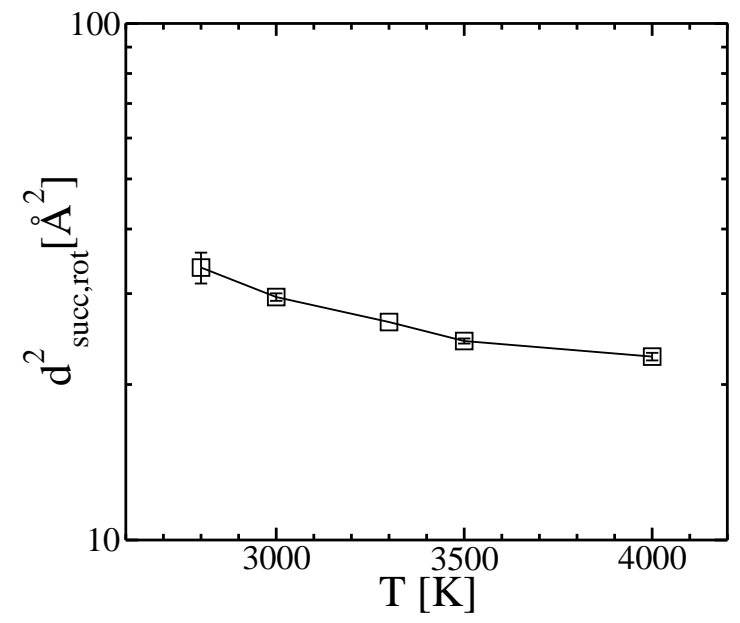

Figure 5: Temperature dependence of $d_{\text {succ,rot }}^{2}$ (see definition in text).

temperature-independent for the range of temperatures, analyzed in this work. Apart from the temperatureindependence one observes a surprising similarity of $d_{r o t, O}^{2}$ and $d_{\text {trans }, O}^{2}$. This implies that, maybe in contrast to expectation, the nature of the oxygen dynamics in translational time intervals is similar to that in rotational time intervals.

\section{Transitions between rotational states}

In the final part of this section we discuss some properties of the transitions between rotational states. These results will shed some light on the relation between the PEL-properties and the actual dynamics, as will be discussed in Sect.VI.

First, we analyze the typical distance in the configuration space between successive rotational states. For this purpose we define

$$
d_{\text {succ }, r o t, O}^{2} \equiv \frac{\sum_{i} d_{i}^{2}}{n_{\text {succ }, \text { rot }}}
$$

where $n_{\text {succ,rot }}$ denotes the number of transition between different rotational states, added over all rotational periods. Its temperature dependence is shown in Fig [5] Upon cooling $d_{\text {succ,rot,O }}^{2}$ increases. This means that the typical distance between subsequent rotational states becomes larger for lower temperatures, i.e. more complex oxygen permutations occur. As an immediate consequence on average more MB transitions are necessary at lower temperatures to reach a new rotational state within the same rotational period.

More detailed insight into the rotational dynamics can be obtained by analyzing the distribution of the mean square displacement between two successive rotational states (here: $T=3000 \mathrm{~K}$ ). This distribution is shown in Fig 6 One can clearly see that most displacements are around $20 \AA^{2}$ which corresponds to a simple $\mathrm{C}_{3}$ rotation.

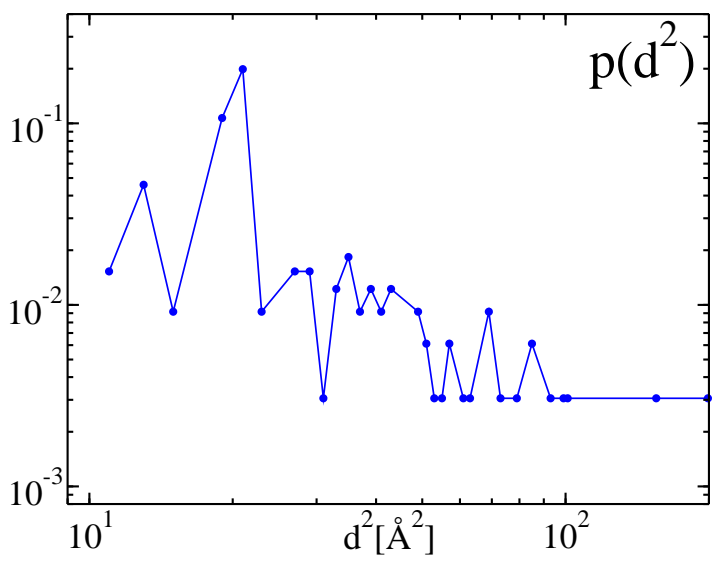

Figure 6: Top: The distribution of the distances between successive rotational states at $T=3000 \mathrm{~K}$ as identified during our MD simulations. Note the logarithmic scale.

A second but significantly smaller peak at approx. 13 $\AA^{2}$ reflects the exchange of two adjacent oxygen atoms. Furthermore, a very small fraction of more complex permutations with larger displacements is observed.

\section{QUANTIFICATION OF THE DECOUPLING OF OXYGEN AND SILICON DYNAMICS}

In this section we first analyze the ratio of the oxygen diffusion constant to the silicon diffusion constant $D_{O}(T) / D_{S i}(T)$. Its temperature dependence, as obtained from our molecular dynamics simulations, is shown in Fig 7 Included are also data from the previous MD work by Horbach et al [5] and by Saika-Voivod et al [13]. Furthermore we checked for two temperatures that for our system parameters the ratio does not depend on the system size within statistical accuracy. Note that the mild variations of this ratio for different simulations may be due to the slightly different densities or the different cutoff conditions for the pair-potentials. In any event, on a semi-quantitative level all simulations display a very similar increase of $D_{O}(T) / D_{S i}(T)$ with decreasing temperature.

Using the quantities, introduced in the previous section, we may write

$$
\begin{aligned}
\frac{D_{O}(T)}{D_{S i}(T)} & =\frac{n_{\text {trans }}(T) \cdot d_{\text {trans }, O}^{2}+n_{\text {rot }}(T) \cdot d_{\text {rot }, O}^{2}}{2 \cdot n_{\text {trans }}(T) \cdot d_{\text {trans }, S i}^{2}}, \\
& =\frac{d_{\text {trans }, O}^{2}(T)}{2 d_{\text {trans }, S i}^{2}}+\frac{n_{\text {rot }}(T) \cdot d_{\text {rot }, O}^{2}}{2 n_{\text {trans }} \cdot d_{\text {trans }, S i}^{2}}
\end{aligned}
$$

The factor of 2 reflects the stoichiometry of $\mathrm{SiO}_{2}$. Using

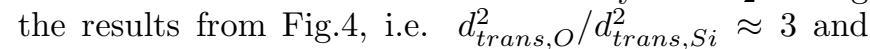
$d_{\text {rot }, O}^{2} \approx d_{\text {trans }, O}^{2}$, the ratio $D_{O} / D_{S i}$ can be rewritten as 


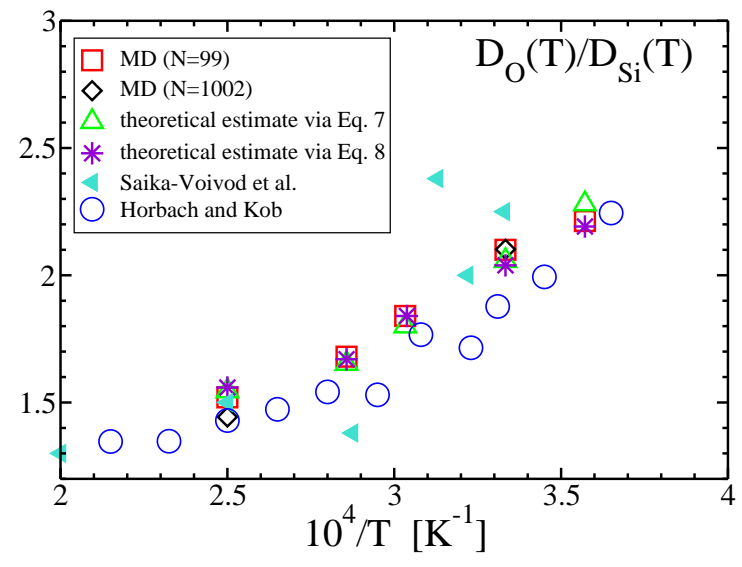

Figure 7: The decoupling between oxygen and silicon dynamics. Shown is the temperature dependence of the ratio of the oxygen diffusion constant to the silicon diffusion constant $D_{O} / D_{S i}$ as well as the theoretical estimations from Eq.7 and Eq.8. Moreover, the corresponding values from literature [5, 13] are included.

$$
\frac{D_{O}(T)}{D_{S i}(T)} \approx \frac{3}{2} \cdot\left(1+\frac{n_{\text {rot }}(T)}{n_{\text {trans }}(T)}\right)
$$

This result quantifies the general statement, formulated above, that the decoupling is exclusively related to the increasing relevance of rotations at lower temperatures, as expressed by the temperature dependence of $n_{\text {rot }}(T) / n_{\text {trans }}(T)$. The estimated values of $D_{O}(T) / D_{S i}(T)$ according to Eq 7 are also included in Fig[7] The excellent agreement with the simulated $D_{O}(T) / D_{S i}(T)$ constitutes a consistency check of the analysis of Sect.III.

\section{ENERGETIC ASPECTS OF MB TRANSITIONS}

MBs are mainly characterized by energy and residence time. In literature it has been shown that the energy plays an important role for the dynamics [23, 27]. Thus, one may expect that energy also affects the nature of the MB transitions in the present context. In order to check the energy dependence for the rotational and translational transitions, we determine the number of MB transitions in the rotational periods $n_{\text {rot }}(e, T)$ and the translational periods $n_{\text {trans }}(e, T)$ in dependence on $\mathrm{MB}$ energy e. From $\operatorname{Eq}\left[7 D_{S i}(T) / D_{O}(T)\right.$ can be written as

$$
\begin{aligned}
\frac{D_{S i}(T)}{D_{O}(T)} & =\frac{2}{3} \frac{n_{\text {trans }}(T)}{n_{\text {trans }}(T)+n_{\text {rot }}(T)} \\
& =\frac{2}{3} \int \operatorname{de} \varphi(e, T) \cdot P_{\text {trans }}(e, T)
\end{aligned}
$$

$\varphi(e, T)$ has been defined in $\mathrm{Eq} 2$ and denotes the probability that a MB has the energy $e$. Furthermore,
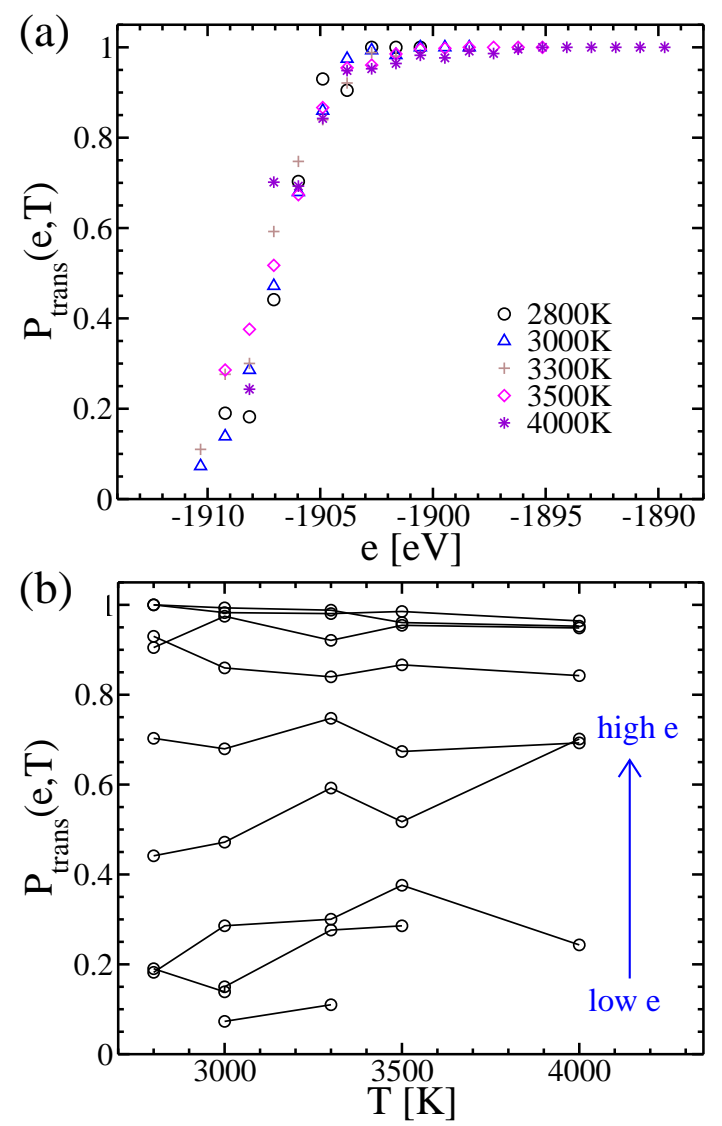

Figure 8: Energy (a) and temperature (b) dependence of the probability of translational relaxations $P_{\text {trans }}(e, T)$.

$P_{\text {trans }}(e, T)$ is the probability that a MB of energy $e$ is visited during a translational period. A small value of $P_{\text {trans }}(e, T)$ thus implies that after leaving a MB of energy $e$ the system very likely will later on end up in a rotational state. The energy and temperature dependence of $P_{\text {trans }}(e, T)$ is shown in Figs 8 (a) and (b), respectively. Most importantly, $P_{\text {trans }}(e, T)$ depends strongly on energy whereas it does not show any significant temperature dependence. In Fig 8(a) one can separate the energy dependence of $P_{\text {trans }}(e, T)$ into two regimes. In the high-energy regime one has $P_{\text {trans }}(e, T) \approx 1$. This means that no significant rotational processes happen for highenergy MB. When the MB energy decreases, rotational processes become relevant. This results in a decrease of $P_{\text {trans }}(e, T)$. Interestingly, more than $90 \%$ of the transitions involving $\mathrm{MB}$ with energies close to the cutoff energy $e_{c}$ contribute to the rotational dynamics. From the present results we have to conclude that energy not only determines relaxation rates as shown in the previous studies [23, 27] but also determines the relevance of rotational processes.

As a consistent check, we calculate $D_{O}(T) / D_{S i}(T)$ via Eq. 8 The results are also included in Fig[7 Naturally, a good agreement with the actually observed $D_{O}(T) / D_{S i}(T)$ is found. 


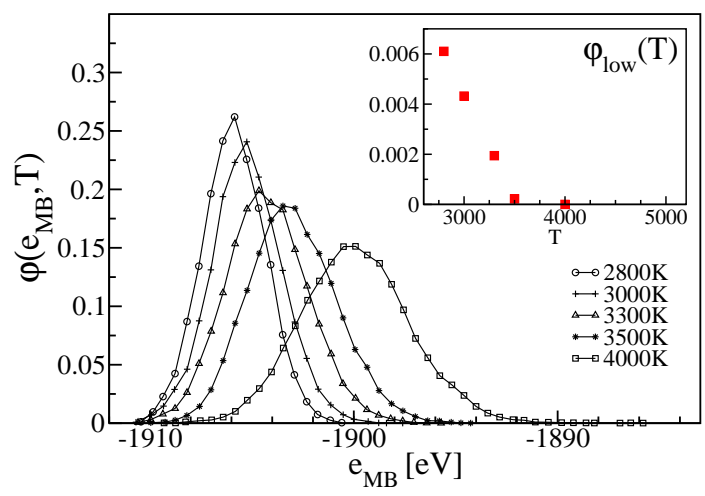

Figure 9: The distribution $\varphi(e, T)$ of MB for different temperatures. In the inset the temperature dependence of $\varphi_{\text {low }}=$ $\int_{e_{c}}^{e_{c}+1 e V} \operatorname{de} \varphi(e, T)$ is shown. It reflects the number of lowenergy MB close to the cutoff of the PEL.

\section{DISCUSSION AND SUMMARY}

In previous work on silica we were able to express the oxygen diffusion constant via

$$
D_{O}(T) \propto \int \operatorname{dep}(e, T) /\langle\tau(e, T)\rangle .
$$

where the average waiting time $\langle\tau(e, T)\rangle$ can be written as

$$
\langle\tau(e, T)\rangle=\tau_{0}(e) \exp (\beta V(e))
$$

with an energy-dependent attempt frequency $1 / \tau_{0}(e)$ and an effective barrier height $V(e)$.

This set of equations implies that from the three temperature-independent quantities $G(e), \tau_{0}(e)$ and $V(e)$ the thermodynamics as well as the temperature dependence of the oxygen dynamics (in terms of the diffusion constant) can be predicted. In particular $V(e)$ can be rationalized in terms of the local topology of the PEL 23]. This implies a basically complete understanding of the oxygen diffusion in terms of properties of the PEL. In this work we have identified a further (nearly) temperatureindependent function $P_{\text {trans }}(e)$ which allows one to predict the enhanced slowing-down of silicon diffusion as compared to oxygen diffusion.

In the following we will argue that the results, obtained so far, are compatible with the general physical scenario of relaxation processes in silica, as formulated in [23]. As a main result it has been shown that there exists a crossover-energy $e_{\text {cross }} \equiv-1905 \mathrm{eV}$ which separates two dynamical regimes. For $e>e_{\text {cross }}$ the escape dynamics is non-activated whereas for significantly lower $e$ the system first has to climb up in the PEL to IS with energies close to $e_{\text {cross }}$ until it can effectively escape from this region of configuration space. On a qualitative level this observation can be related to the strong energy dependence of the density of $\mathrm{MB} G(e)$ or $\varphi(e, T)$, respectively. In the considered range of temperatures much less than $1 \%$ of all configurations possess an energy less than $e_{c}+1 \mathrm{eV}$; see Fig 9] In contrast, nearly every second MB has an energy less than $e_{\text {cross. }}$. As a consequence for MB with $e>e_{\text {cross }}$ the PEL supplies many adjacent configurations of similar energy with connecting saddles of at most $1 \mathrm{eV}$ which the system may easily reach, giving rise to non-activated dynamics whereas in the low-energy limit this is no longer possible due to the lack of such configurations.

For rationalizing the features, observed in this work, we discuss in qualitative terms the distribution of $\mathrm{MB}$ in configuration space relative to a given $\mathrm{MB}$ (denoted central $\mathrm{MB}$ ) with energy $e_{0}$, visited at a given temperature $T$. We introduce the function $\varphi_{\text {all }}\left(e, d^{2} \mid e_{0}\right)$ as the probability that a randomly selected MB is a distance $d^{2}$ away from the central $\mathrm{MB}$ and has an energy $e$. This probability distribution has two contributions

$$
\varphi_{\text {all }}\left(e, d^{2} \mid e_{0}\right)=\varphi_{\text {rot }}\left(e, d^{2} \mid e_{0}\right)+\varphi_{\text {rest }}\left(e, d^{2} \mid e_{0}\right) .
$$

The first contribution takes into account configurations which result from oxygen permutations of the central MB. This distribution can be written as $\varphi_{\text {rot }}\left(e, d^{2} \mid e_{0}\right) \propto$ $G_{\text {rot }}\left(d^{2}\right) \delta\left(e-e_{0}\right)$ where $G_{\text {rot }}\left(d^{2}\right)$ is the distribution of distances, when taking into account all possible 66 ! permutations. We have calculated $G_{\text {rot }}\left(d^{2}\right)$ for one representative low-energy configuration and have exchanged the indices of the oxygen atoms via Monte-Carlo techniques. To determine the distribution over many orders of magnitude we have used appropriately adjusted flat-histogram techniques [29]. The resulting distribution $G_{r o t}\left(d^{2}\right)$ is shown in Fig[10] One can clearly see a few peaks for smaller distances and a transition to a continuous behavior for larger distances. Naturally, the $d^{2}$-values of the peaks agree with those observed during the actual transitions between rotational states. For $d^{2} \geq 100 \AA^{2}$ this distribution no longer shows any wiggles. This reflects the fact that a transition with a large value of $d^{2}$ can be achieved by a multitude of different oxygen permutations. In contrast, for small $d^{2}$ discrete distances can be identified which belong to specific types of reorientations (like $C_{3}$ rotations). Comparison with the actually observed transitions in Fig [6] shows that in particular more complex permutations, related to larger values of $d^{2}$, are strongly suppressed. Interestingly, also exchange processes of adjacent oxygen atoms, corresponding to the first peak, are significantly suppressed.

The second contribution $\varphi_{\text {rest }}\left(e, d^{2} \mid e_{0}\right)$ is related to the remaining $\mathrm{MB}$ which have a different structure as the central MB. For sufficiently large $d^{2}$ one may naturally write $\varphi_{\text {rest }}\left(e, d^{2} \mid e_{0}\right) \propto G_{\text {rest }}\left(d^{2}\right) \varphi(e, T)$ with $\varphi(e, T)$ defined in EqL2 Qualitatively, this means that for regions sufficiently far away from the central MB the distribution of $\mathrm{MB}$ is not influenced by the presence of a MB with $e=e_{0}$ at the origin. One expects that for large $d^{2}$ the distribution $G_{\text {rest }}\left(d^{2}\right)$ increases much faster than $G_{\text {rot }}\left(d^{2}\right)$, i.e. with a larger exponent of $d^{2}$, because $G_{\text {rest }}\left(d^{2}\right)$ contains the exponentially larger number of different IS as well their permutations (except for those taken into account by $\left.G_{r o t}\left(d^{2}\right)\right)$. Thus, for large $d^{2}$ 


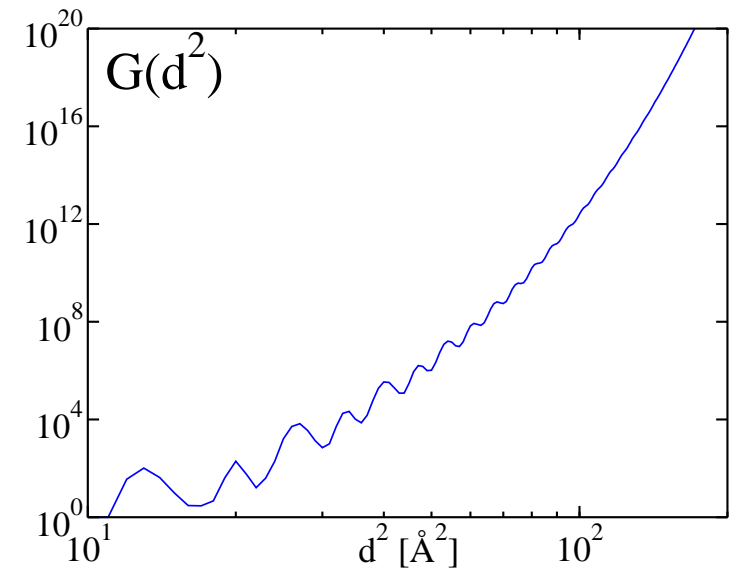

Figure 10: The distribution $G_{r o t}\left(d^{2}\right)$ of all distances between configurations with oxygen permutations, as obtained from Monte-Carlo simulations.

one expects $G_{r o t}\left(d^{2}\right) \ll G_{r e s t}\left(d^{2}\right)$. This dramatic difference may disappear for small $d^{2}$ because the ratio of both functions becomes smaller for smaller $d^{2}$. Furthermore, in contrast to the oxygen permutations the possible transitions to adjacent MB are more continuously distributed with respect to their distances $d^{2}$. As a consequence the continuous regime of $G_{\text {rest }}\left(d^{2}\right)$ will probably extend to much smaller values of $d^{2}$ than observed for $G_{\text {rot }}\left(d^{2}\right)$, i.e. approx. $100 \AA^{2}$. Thus, one may speculate that for small $d^{2}$ (e.g. $\left.d^{2} \approx 20 \AA^{2}\right) G_{\text {rot }}\left(d^{2}\right)$ is of the same order as $G_{\text {rest }}\left(d^{2}\right)$. Of course, for a more quantitative characterization one directly has to determine $G_{\text {rest }}\left(d^{2}\right)$, which, however, is a formidable task.

We note in passing that the factorization of $\varphi_{\text {rest }}\left(e, d^{2} \mid e_{0}\right) \propto G_{\text {rest }}\left(d^{2}\right) \varphi(e, T)$ will break down for $d^{2} \rightarrow 0$, as known from the study of the tunneling systems [30]. There it turned out that for very nearby IS $\left(d^{2} \leq 1 \AA^{2}\right)$ the energy difference does not depend on $e_{0}$ which implies that $\varphi_{\text {rest }}\left(e, d^{2} \mid e_{0}\right)$ is a function of $e-e_{0}$.

With the general concepts, introduced above, the following observations have to be rationalized: (i) The presence of rotations with small values of $d^{2}$; see Fig. 6 but the strong suppression of rotations with larger $d^{2}$. (ii) The additional suppression of particle exchange with $d^{2} \approx 13 \AA^{2}$. (iii) The relevance of rotations only for $e<e_{\text {cross }}$ and the increase of rotations for decreasing energy. (iv) The increase of $d_{\text {succ,rot }, O}^{2}$ with decreasing temperature. (v) The temperature-independence of $P_{\text {trans }}(e)$. (vi) The similarity of $d_{\text {rot }, O}^{2}$ and $d_{\text {trans }, O}^{2}$.

(i) For large $d^{2}$ the function $G_{r e s t}\left(d^{2}\right)$ will be orders of magnitude larger than $G_{\text {rot }}\left(d^{2}\right)$. Thus for purely statistical reasons hardly any permutations with large $d^{2}$ are observed; see Fig [ In this context it is surprising that nevertheless a very small number of permutations with $d^{2}$ up to $80 \AA^{2}$ are observed which from this purely statistical consideration should be extremely unlikely. Maybe there are specific pathways on the PEL which enhance the probability of specific permutations. (ii) For oxygen ex- change processes massive reorientations are necessary in contrast to, e.g., effective $C_{3}$ rotations. To perform an exchange process the system would have to reach regions of the configuration space which are far away from the central MB. As a consequence, for purely statistical reasons the probability to complete the exchange process is very small. (iii) As discussed above for small $d^{2}$ the function $G_{\text {rot }}\left(d^{2}\right)$ may be of the same order as $G_{\text {rest }}\left(d^{2}\right)$. Furthermore, $\varphi_{\text {rest }}\left(e, d^{2} \mid e_{0}\right) \propto \varphi(e, T)$ is very small for $e \approx e_{c}$, see Fig 9 implying $\varphi_{\text {all }}\left(e_{0}, d^{2} \mid e_{0}\right) \approx G_{\text {rot }}\left(d^{2}\right) \varphi_{\text {rot }}\left(e_{0}, d^{2}\right)$ for $e_{0} \approx e_{c}$. Thus, for statistical reasons the relevance of rotational states is significantly enhanced for very low energies. In contrast, for $e_{0} \geq e_{\text {cross }}$ the system possesses a large number of adjacent IS with similar energy such that again for statistical reasons the probability to reach one of the rotational states is very small. (iv) As a further consequence the range of distances for which the probability to find a rotational state is non-negligible increases with decreasing energy. Thus, in agreement with observation one would expect an increase of the average distance between successive rotational states with decreasing energy and thus with decreasing temperature. (v) As discussed above for the transition from one lowenergy configuration $\left(e_{0} \approx e_{c}\right)$ to a new low-energy configuration the system first has to climb up the PEL to energies close to $e_{\text {cross }}$. This trajectory basically forms an energy-upwards path without major intermediate barriers 23]. In analogy, the subsequent path to a new lowenergy configuration is not expected to possess significant barriers, in similarity to the funnel-like picture of protein folding. Stated differently, the search for the subsequent low-energy configuration is basically entropy-driven so that $P_{\text {trans }}$, indeed, should not significantly depend on temperature. (vi) Whether or not the system ends up in a rotational state is mainly a question of the statistical availability of these states. As a consequence one would expect that the nature of the oxygen dynamics in translational and rotational periods should not differ too much. This is consistent with the observed similarity of $d_{r o t, O}^{2}$ and $d_{\text {trans }, O}^{2}$.

According to $\mathrm{Eq} 8$ the fraction of the rotational and translational dynamics is related to the thermodynamic weighting of the $\mathrm{MB}$ population, $\varphi(e, T)$. Therefore, the temperature dependence of $n_{\text {trans }}(T) / n_{M B}(T)$ presented above is just a consequence of the temperature dependence of $\varphi(e, T)$. It is interesting to estimate the value of $D_{O} / D_{S i}$ at the glass transition temperature $T_{g}(1450 \mathrm{~K})$. From the density of MBs $G(e)$ and waiting time $\langle\tau(e, T)\rangle$ one can predict $\varphi(e, T)$ at $T_{g}$. By using $\mathrm{Eq} \nabla$ the estimated value for $D_{O} / D_{S i}$ at $T_{g}$ is of the order of $10^{1}$, which is one order of magnitude smaller than the experimental value of $\approx 10^{2}[2,3]$. This discrepancy may be resolved in different ways: (i) At lower temperatures further decoupling mechanisms set in. (ii) The temperatureindependence of $P_{\text {trans }}$ no longer holds for temperatures closer to the calorimetric glass transition. (iii) The limits of the BKS-potential to predict the details of the oxygen and silicon dynamics in silica are reached. 
The present PEL approach to characterize the dynamics of oxygen and silicon allows one to obtain a relatively simple physical picture about the oxygen dynamics as well as the silicon dynamics. The essential key ingredient to understand the onset of the decoupling of oxygen and silicon diffusion is the presence of rotational dynamics which only contributes to the oxygen dynamics and which can be rationalized in simple statistical terms in the framework of the PEL approach. Excluding the rotational periods, the difference in activation energies of silicon and oxygen disappears in the considered range of temperatures.

We would like to acknowledge discussions with M. Vogel on this subject and the NRW Graduate School of Chemistry and the DFG (SFB 458) for the support of this work.
[1] C. A. Angell, J. Non-Cryst. Solids. 131-133, 13 (1991).

[2] J. C. Mikkelsen, Appl. Phys. Lett. 45, 1187 (1984).

[3] G. Brebec, R. Seguin, C. Sella, J. Bevenot, and J. Martin, Acta. Metall. 28, 327 (2003).

[4] M.L.F. Nascimento and E.D. Zanotto, Phys. Rev. B. 73, 024209 (2006).

[5] J. Horbach and W. Kob, Phys. Rev. B. 60, 3169 (1999).

[6] B. W. H. van Beest, G. J. Kramer, and R. A. van Santen, Phys. Rev. Lett. 64, 1955 (1990).

[7] M. Vogel and S. C. Glotzer, Phys. Rev. Lett. 92, 255901 (2004).

[8] V. Teboul, A. Monteil, F. L.C., A. Kerrache, and S. Maabau, Eur. Phys. J. B 40, 49 (2004).

[9] C. Donati, J. F. Douglas, W. Kob, S. J. Plimpton, P. H. Poole, and S. C. Glotzer, Phys. Rev. Lett 80, 2338 (1998).

[10] M. S. Shell, P. G. Debenedetti, and A. Z. Panagiotopoulos, Phys. Rev. E 66, 011202 (2002).

[11] T. F. Middleton and D. J. Wales, Phys. Rev. B 64, 024205 (2001).

[12] B. Doliwa and A. Heuer, Phys. Rev. E. 67, 030501 (2003).

[13] I. Saika-Voivod, P. H. Poole, and F. Sciortino, Phil. Mag. 48, 1437 (2004).

[14] F. H. Stillinger and T. A. Weber, Phys. Rev. A 25, 978 (1982).

[15] S. Sastry, P. G. Debenedetti, and F. H. Stillinger, Nature 393, 554 (1998).

[16] F. Sciortino, J of Stat. Mech. 83, P05015 (2005).
[17] I. Saika-Voivod, F. Sciortino, and P. H. Poole, Phys. Rev. E. 69, 041503/1 (2004).

[18] D. J. Wales, Energy landscapes (Cambridge University Press, 2003).

[19] M. Goldstein., J. Chem. Phys. 51, 3728 (1969).

[20] E. La Nave, H. Stanley, and F. Sciortino (2002).

[21] B. Doliwa and A. Heuer, J. Phys. C: Cond. Mat. 15, S849 (2003).

[22] A. Saksaengwijit, J. Reinisch, and A. Heuer, Phys. Rev. Lett. 93, 235701 (2004).

[23] A. Saksaengwijit and A. Heuer, Phys. Rev. E. 33, 061503 (2006).

[24] L. Martin-Samos, Y. Limoge, J. Crocombette, G. Roma, and N. Richard, Phys. Rev. B 71, 014116 (2005).

[25] N. Mousseau and G. T. Barkema, J Chem. Phys. 112, 960 (2000).

[26] J. Reinisch and A. Heuer, Phys. Rev. Lett. 95, 155502 (2005).

[27] B. Doliwa and A. Heuer, Phys. Rev. E. 67, 031506 (2003).

[28] In a strict sense $G(e)$ is the density of states weighted by the harmonic contributions; see Ref.12 for a closer disucssion. For silica, however, this additional contribution is very small.

[29] M. S. Shell, P. G. Debenedetti, and A. Z. Panagiotopoulos, J. Phys. Chem. B 108, 19748 (2004).

[30] J. Reinisch and A. Heuer, Phys. Rev. B 70, 064201 (2004). 